\title{
Wie man Protokollverletzungen in der Komplementärmedizin richtig behandelt
}

\author{
Rainer Lüdtke \\ Karl und Veronica Carstens-Stiftung, Essen, Deutschland
}

Seit über 20 Jahren schon erhitzt der Streit über die Intention-to-treat(ITT)-Analyse Praktiker und Methodiker in der Medizin. Unter ITT versteht man eine Auswertungsstrategie für kontrollierte Studien, in der alle Patienten in der Therapiegruppe ausgewertet werden, der sie ursprünglich, also bei Studienbeginn, zugeordnet waren. Und zwar, und das ist der Knackpunkt, unabhängig davon, ob sie überhaupt die vorgesehene Therapie erhalten haben, ob sie die vorgesehenen Einschlusskriterien erfüllt haben, ob sie die Therapievorgaben vollständig befolgt haben, ob sie unerlaubte Begleitmedikationen erhalten haben (z.B. die Therapie der Vergleichsgruppe) oder ob sie bei Studienabschluss noch verwertbare Daten vorlegen konnten oder nicht.

Für den Kliniker ist diese Strategie nur schwer nachzuvollziehen. Warum sollte ein Patient der Kontrollgruppe, der eine laut Studienprotokoll unerlaubte Begleitmedikation eingenommen hat, dennoch so gewertet werden, als hätte er diese nicht genommen? Wie sollte ein Patient der Verumgruppe, der seine Therapievorgaben nur unzureichend befolgt hat, dazu beitragen können, die Wirksamkeit eben gerade dieser Therapie sinnvoll beurteilen zu können? Ein solches Vorgehen läuft unserem gesunden Menschenverstand zuwider. Es erscheint uns logisch, dass bei der Bewertung einer Therapie nur solche Beobachtungen berücksichtigt werden dürfen, die auf einer realen, nachvollziehbaren und eindeutigen Grundlage beruhen. Es erscheint uns deshalb auch logisch, dass Patienten, die sich nicht protokollgerecht verhalten haben, aus der Auswertung ausgeschlossen werden müssen.

Methodiker dagegen verweisen auf ein primär statistisches Argument: Grundlage jeder statistischen Beurteilung einer Therapie (und damit letztendlich seiner Wirksamkeitsbewertung) sind statistische Schätz- und Testverfahren. Diese sind vor allem dann gültig, wenn die Zuteilung zu Verum- und Kontrollgruppe randomisiert erfolgte, also allein auf Zufallsprinzipien beruhte. Nur dann kann man sich sicher sein, dass keine versteckten Variablen die Beziehung zwischen Therapie und Behandlungserfolg überlagern. Der nachträgliche Ausschluss bereits randomisierter Patienten zerstört jedoch diese Struktur: Nicht allein der Zufall, sondern auch das Verhalten der Patienten während der Studie ist jetzt dafür verantwortlich, wie die Gruppen zusammengesetzt sind. Dadurch entfällt die wichtigste Voraussetzung für die Gültigkeit der verwendeten statistischen Verfahren; versteckte Variablen können die Ergebnisse verfälschen. Ja, eine solche Verfälschung ist sogar sehr wahrscheinlich, da die nachträglich ausgeschlossenen $\mathrm{Pa}-$ tienten in der Regel nicht repräsentativ für die Gesamtzahl aller Patienten sein dürften. Allein die Einnahme von Begleitmedikationen kann auf eine generell schlechtere Prognose dieser Patienten hindeuten. Andere Patienten bleiben einer Follow-up-Untersuchung fern, weil sich ihre Erkrankung akut verschlimmert hat. Wieder andere können eventuell ein Therapieschema aufgrund von Nebenwirkungen nicht befolgen.

So weit die Theorie. Doch hat diese auch Relevanz für die Praxis? Macht es also wirklich einen Unterschied, ob eine Studie nach dem ITT-Prinzip ausgewertet wurde oder nicht? Wenn man der Schweizer Arbeitsgruppe um P. Jüni (übrigens Mitbegründer von Forschende KomplementÄrmedizin) und E. Nüesch folgt, dann heißt die Antwort Nein. Eine systematische Über- oder Unterschätzung der Wirksamkeit der Prüftherapie erfolgt nicht [1]. Meist nicht, denn es gibt eine Ausnahme ...

Die Schweizer nahmen 14 Metaanalysen zur Wirksamkeit verschiedener medizinischer Interventionen zur Schmerzbehandlung bei Cox- oder Gonarthrose unter die Lupe. Diese Metaanalysen berichteten von 167 randomisierten klinischen Studien, von denen 39 nach dem ITT-Prinzip ausgewertet wurden, 129 aber aus verschiedenen Gründen zum Teil erhebliche Patientenzahlen ausgeschlossen hatten. 11 der 14 Metaanalysen berichteten von signifikanten Effekten zugunsten der untersuchten Intervention. Die größten Effekte wurden für transkutane elektrische Nervenstimulation (TENS), Glucosamin, Chondroitin und Akupunktur gefunden, also für

\section{KARGER}

Fax +497614520714

Information@Karger.de

www.karger.com (c) 2009 S. Karger GmbH, Freiburg

Accessible online at:

www.karger.com/fok
Dipl.-Stat. Rainer Lüdtke

Karl und Veronica Carstens-Stiftung

Am Deimelsberg 36

45276 Essen, Deutschland

Tel. +49 20156305-16, Fax -30

r.luedtke@carstens-stiftung.de 
typische Therapien der Komplementärmedizin (CAM) - zumindest wurden sie von den Autoren als solche eingestuft. Gleichzeitig zählten diese vier Therapien zu denjenigen, bei denen die Studienlage besonders heterogen war, die Effekte sich also von Studie zu Studie besonders stark unterschieden, was natürlich die Hypothese nahelegt, dass es hier einen Zusammenhang gibt.

Und $a b$ da wird es richtig interessant. Die Schweizer Forscher konnten nämlich zeigen, dass in den Metaanalysen zur CAM (neben den vier oben genannten zählte man auch noch die niedrigenergetische Lasertherapie dazu) der Therapieeffekt wesentlich größer eingeschätzt wurde, wenn die Studie nicht nach dem ITT-Prinzip ausgewertet wurde. Eine Aussage, die für konventionelle Therapien (unter anderem topische und orale nichtsteroidale Antirheumatika (NSAR), Paracetamol, Gewichtsreduktion und Bewegungstherapie) so nicht gilt: Hier waren die Studienergebnisse unabhängig davon, ob Patienten aus der Auswertung ausgeschlossen wurden oder nicht. - Ein Befund, der CAM-Interessierte, Praktiker wie Methodiker, aufhorchen lassen sollte. Wir sprechen hier nämlich nicht von irgendwelchen vernachlässigbaren Unterschieden, sondern von relevanten Effekten. Nur als Beispiel: Der mittlere standardisierte schmerzlindernde Effekt einer Akupunkturbehandlung liegt bei 0,49. (Zum Vergleich: der Effekt oraler NSARs beträgt 0,40 , ist also etwas niedriger.) Ein Großteil dieses Effekts ist auf die Auswertungsstrategie zurückzuführen: Werden die Akupunkturstudien nach dem ITT-Prinzip ausgewertet, dann ist der geschätzte Effekt um 0,36 kleiner, als wenn dieses Prinzip verletzt wird. Mit anderen Worten: Wären alle Studien nach dem ITT-Prinzip ausgewertet worden, wäre der standardisierte schmerzlindernde Effekt der Akupunktur wesentlich geringer als 0,49 eingeschätzt worden. Bei Chondroitin ist die Lage noch dramatischer: Der Gesamteffekt wird auf 0,72 geschätzt, der Unterschied zwischen gemäß ITT und nicht gemäß ITT ausgewerteten Studien beträgt 0,82. Es bliebe also nicht mehr viel vom Chondroitin-Effekt übrig, wenn alle Studien dem ITT-Prinzip gefolgt wären.

Die Schlussfolgerung aus diesen Daten ist aus meiner Sicht einfach: Gerade in der CAM-Forschung müssen wir uns bewusst sein, dass das Ausschließen von Patienten aus der Studienauswertung ein sehr hohes Bias-Risiko birgt. Studien, die Patienten wegen Nicht-Erscheinens zu Follow-ups, NonCompliance oder der unerlaubten Einnahme von Begleitmedikationen ausschließen, überschätzen den tatsächlichen The- rapieeffekt möglicherweise dramatisch und sind daher wenig glaubwürdig.

Viel schwieriger scheint mir die Frage danach, warum eigentlich diese Effekte nur in der Komplementärmedizin auftreten. Meine Vermutung: Vor allem die hohen Erwartungen, die CAM-Patienten an ihre Therapie haben, spielen eine Rolle. Werden diese Erwartungen nicht oder nur teilweise erfüllt, so steigt das Risiko von Protokollverletzungen. Es ist nicht abwegig anzunehmen, dass dieses Risiko in der Komplementärmedizin deutlich höher ist als in der konventionellen Medizin. Dafür spricht, dass in einigen Studien tatsächlich die Erwartungen an eine CAM-Therapie deutlich höher waren als an eine konventionelle Vergleichstherapie, z.B. an eine Blutegeltherapie im Vergleich zu topischen NSARs bei Daumengelenksarthrose [2]. Zudem gibt es nichtverblindete Studien, in denen die Patientenpräferenz so groß war, dass sich ein Großteil der Patienten schon weigerte, die zufällig per Los zugewiesene, aber ungeliebte Therapie zu beginnen [3], was im Fall der Akupunktur bei Kopfschmerzen sogar dazu geführt hat, dass die Rekrutierung in die konventionelle Vergleichsgruppe gestoppt werden musste [4].

Manchmal scheint mir, dass wir uns in der Komplementärmedizin einfach schon zu sehr an übersteigerte Patientenerwartungen und hohe Raten von Therapieabbrechern gewöhnt haben, um diese noch als Problem wahrzunehmen. Ernst et al. [5] zum Beispiel fanden Compliance-Raten von $60 \%$ in der Mind-Body-Medicine durchaus akzeptabel.

Übrigens: Wenn die Hypothese mit den hohen Patientenerwartungen stimmt, dann hat das natürlich auch einen direkten Einfluss auf jeden einzelnen CAM-Praktiker und seine/ihre Bewertung der eigenen Therapie. Der CAM-Praktiker kann per definitionem - seine Heilungserfolge immer nur an einem selektierten Patientengut ablesen, nämlich den Patienten, die seine Praxis mindestens ein zweites Mal aufsuchen. Was dann aber bei übersteigerten Patientenerwartungen und geringer Compliance nichts anderes heißt, als dass CAM-Praktiker viel eher dazu neigen werden, ihre Erfolge zu überschätzen als ihre konventionellen Kollegen.

Nüeschs Artikel sollte also von allen, Praktikern wie Methodikern, ernst genommen werden. Eine Diskussion über das ITT-Prinzip und seine Folgen, gerade in CAM, erscheint mir zwingend notwendig, auch wenn der Artikel sich nur mit einigen CAM-Formen und nur einem eingeschränkten Krankheitsbild beschäftigt.

\section{Literatur}

1 Nüesch E, Trelle S, Reichenbach S, Rutjes AW, Bürgi E, Scherer M, Altman DG, Jüni P: The effects of excluding patients from the analysis in randomised controlled trials: meta-epidemiological study. BMJ 2009;339:b3244.

$\checkmark 2$ Michalsen A, Lüdtke R, Cesur O, Afra D, Musia F, Baecker M, Fink M, Dobos GJ: Effectiveness of leech therapy in women with symptomatic arthrosis of the first carpometacarpal joint: A randomized controlled trial. Pain 2008;137:451-459.
3 Bernateck M, Becker M, Schwake C, Hoy L, Passie T, Parlesak A, Fischer MJ, Fink M, Karst M: Adjuvant auricular electroacupuncture and autogenic training in rheumatoid arthritis: a randomized controlled trial. Auricular acupuncture and autogenic training in rheumatoid arthritis. Forsch Komplementmed 2008;15:187-193.
-4 Endres HG, Bowing G, Diener HC, Lange S, Maier C, Molsberger A, Zenz M, Vickers AJ, Tegenthoff M: Acupuncture for tension-type headache: a multicentre, sham-controlled, patient-and observer-blinded, randomised trial. J Headache Pain 2007;8:306-314.

5 Ernst S, Welke J, Heintze C, Gabriel R, Zollner A Kiehne S, Schwantes U, Esch T: Effects of mindfulness-based stress reduction on quality of life in nursing home residents: a feasibility study. Forsch Komplementmed 2008;15:74-81. 